\title{
High PEEP levels during CPR improve ventilation without deleterious hemodynamic effects in pigs
}

\section{Miriam Renz}

University Medical Center of the Johannes Gutenberg University

\section{Leah Müllejans}

University Medical Center of the Johannes Gutenberg University

Julian Riedel

University Medical Center of the Johannes Gutenberg University

Katja Mohnke

University Medical Center of the Johannes Gutenberg University

\section{René Rissel}

University Medical Center of the Johannes Gutenberg University

Alexander Ziebart

University Medical Center of the Johannes Gutenberg University

Erik Kristoffer Hartmann

University Medical Center of the Johannes Gutenberg University

Robert Ruemmler ( $\boldsymbol{\nabla}$ Robert.ruemmler@unimedizin-mainz.de )

University Medical Center of the Johannes Gutenberg University

\section{Research Article}

Keywords: Resuscitation, PEEP, Porcine, Ventilation, MIGET, EIT

Posted Date: February 16th, 2022

DOI: https://doi.org/10.21203/rs.3.rs-1351659/v1

License: (c) (1) This work is licensed under a Creative Commons Attribution 4.0 International License.

Read Full License 


\section{Abstract}

Background: Invasive ventilation during cardiopulmonary resuscitation (CPR) is very complex due to unique thoracic pressure conditions. Current guidelines do not provide specific recommendations for ventilation during ongoing chest compressions. This trial examines the cardiopulmonary effects of PEEP application during CPR. Three PEEP levels were compared in high (IPPV) and ultralow tidal volume ventilation (ULTVV).

Materials and methods: Forty-two German landrace pigs were anesthetized, instrumented, and randomized into six intervention groups: PEEP 0, 8, and 16 mbar with IPPV or ULTVV. After the induction of ventricular fibrillation, mechanical chest compressions and ventilation were initiated. Blood gases, micropore membrane inlet mass spectrometry facilitated multiple inert gas elimination technique (MMIMS-MIGET) samples and electrical impedance tomography (EIT) loops were taken. Ventilation pressures and hemodynamic parameters were measured continuously. Postmortem lung tissue damage was assessed using the diffuse alveolar damage (DAD) score. Statistical analyses were performed using SPSS, and $p$ values $<0.05$ were considered significant.

Results: The driving pressure $\left(\mathrm{P}_{\text {drive }}\right)$ showed significantly lower values when using PEEP 16 mbar than when using PEEP 8 mbar $(p=0.045)$ or PEEP 0 mbar $(p<0.001)$ when adjusted for the ventilation mode. When not using PEEP, considerable overall lung damage was detected in the PEEP 0 mbar group (vs. PEEP 8 mbar, $p=0.038$; vs. PEEP 16 mbar, $p=0.009$ ) when adjusted for the ventilation mode. No significant differences between the groups occurred in the mean arterial pressure, even when using high PEEP levels.

Conclusion: The use of PEEP during CPR seems beneficial because it optimizes ventilation pressures as well as reduces lung damage without significantly compromising blood pressure. Further studies are needed to examine long-term effects in resuscitated animals.

\section{Background}

In regard to the use of ventilation in cardiac arrest and cardiopulmonary resuscitation (CPR), specific recommendations regarding optimal respiratory settings remain elusive (1). Neither the European Resuscitation Council (ERC) nor the American Heart Association (AHA) provide detailed information on ventilation types or the application of positive end-expiratory pressure (PEEP) $(2,3)$. Guidelines recommend securing the airway during resuscitation and using an endotracheal tube if trained personnel are present. Once the airway is secured, the guidelines suggest using a ventilation rate of 10 breaths per minute and performing continuous chest compressions $(2,3)$. However, it is known that using PEEP during resuscitation can have positive effects on survival (4) and oxygenation (5). When using PEEP during CPR, the improved oxygenation is probably due to the prevention of atelectasis $(4,6,7)$. In cases with increased extrathoracic pressures, the use of PEEP can redistribute ventilation to the dorsal lung regions $(8,9)$. However, the continuous application of PEEP could also lead to increased intrathoracic 
pressures, which can impair venous blood flow $(10,11)$. However, previous studies showed no impaired venous return when applying continuous PEEP during $\operatorname{CPR}(4,6,7)$. Furthermore, the application of PEEP, in general, aligns with lung-protective ventilation strategies that recommend (among others) the application of a PEEP level greater than $5 \mathrm{~cm} \mathrm{H} 2 \mathrm{O}$ (12), although no sufficient data exist to support the clinical relevance during CPR.

In the presented prospective, randomized large animal trial, three different PEEP levels were compared using standard (intermittent positive pressure ventilation, IPPV) and low tidal (ultralow tidal volume ventilation, ULTVV) ventilation modes during CPR. The primary aim of the trial was to examine whether the use of high PEEP levels during CPR can improve gas exchange and optimize ventilation pressures. As a secondary aim, we examined the hemodynamic effects of the applied PEEP levels to determine the clinical value of our findings.

To achieve this, changes in airway pressures were evaluated via ventilation reporting, and pulmonary gas exchange effects were examined via blood gas analysis. Ventilation/perfusion (V/Q) ratios were examined using the MMIMS-MIGET. Changes in lung impedances were analyzed via EIT, and lung tissue damage was evaluated using a validated postmortem histologic scoring system.

\section{Methods}

\section{Anesthesia and Instrumentation}

This animal trial was approved by the State and Institutional Animal Care Committee Rhineland Palatine (approval no. G20-1-065), and all experiments were performed according to the German Animal Protection Law. The trial was planned as a prospective, randomized trial.

Forty-two German landrace pigs (age: 12-16 weeks, weight: 29-34 kg) were examined. Before being transported from a local farm to our Animal Research Facility, the animals were sedated by intramuscular injection of azaperone $(3 \mathrm{mg} / \mathrm{kg})$, ketamine $(1.5 \mathrm{mg} / \mathrm{kg})$ and midazolam $(0.4 \mathrm{mg} / \mathrm{kg})$. Once in our facility, anesthesia was induced directly through an ear cannula by intravenous injection of fentanyl $(4 \mu \mathrm{g} / \mathrm{kg})$, propofol ( $4 \mathrm{mg} / \mathrm{kg})$ and atracurium $(0.5 \mathrm{mg} / \mathrm{kg})$. Anesthesia was maintained via an abdominal venous cannula using continuous infusion of propofol (5-10 mg/kg/h), fentanyl (8-12 $\mu \mathrm{g} / \mathrm{kg} / \mathrm{h})$ and balanced electrolyte infusion $(5 \mathrm{ml} / \mathrm{kg} / \mathrm{h})$.

After orotracheal intubation, mechanical ventilation was performed with an intensive care respirator (Engstroem care station, GE healthcare, Munich) with the following ventilation parameters: tidal volume (Vt) 6-8 $\mathrm{ml} / \mathrm{kg}$, peak inspiratory pressure (Ppeak) $30 \mathrm{mbar}$, positive end-expiratory pressure (PEEP) 5 mbar, and FiO2 0.4. The respiratory rate (RR) was adjusted according to the end-expiratory $\mathrm{CO} 2$ (etCO2) levels with the aim of maintaining them below $6 \mathrm{kPa}(45 \mathrm{mmHg})$. Peripheral oxygen saturation was measured continuously (Masimo Radical 7, Irvine, USA). 
Further preparations included the ultrasound-guided insertion of introducer sheaths (Terumo Europe NV, Leuven, Belgium) into the femoral veins and arteries. A Swan-Ganz catheter (Edwards Lifesciences Services $\mathrm{GmbH}$, Unterschleissheim, Germany) and a fibrillation catheter (VascoMed, Binzen, Germany) were inserted. An electrode belt was placed circularly around the thorax approximately $10 \mathrm{~cm}$ above the diaphragm for electrical impedance tomography measurements (EIT, Pulmo Vista 500, Dräger, Lübeck, Germany). Rectal temperature was continuously measured, and normothermia $\left(37.5-38.5^{\circ} \mathrm{C}\right)$ was maintained by using body surface warming blankets.

\section{Trial protocol and data collection}

A lung recruitment maneuver was conducted after instrumentation. The animals received a fluid bolus of $30 \mathrm{ml} / \mathrm{kg}$ balanced electrolyte solution. A continuous administration $(4 \mathrm{ml} / \mathrm{min})$ of six chemically inert gases with different transpulmonary elimination constants (sulfur hexafluoride, krypton, desflurane, enflurane, diethyl ether, acetone) were dissolved in nontoxic doses in saline for the V/Q ratio measurements. MIGET was performed after a stabilization phase of $30 \mathrm{~min}$ to reach a steady state. A single dose of heparin $(100 \mathrm{lU} / \mathrm{kg})$ was administered before the first measurements were taken.

At the measurement timepoint, baseline healthy (BLH) arterial and central venous blood gases were measured (radiometer, ABL90flex, Denmark), blood samples for the MIGET measurement (MMIMS-MIGET, Oscillogy LLC, Philadelphia, USA) were taken, and EIT recordings were started. Afterward, the animals received a second dose of atracurium $(0.5 \mathrm{mg} / \mathrm{kg})$. The fibrillation catheter was transvenous placed into the right atrium, and continuous ventricular fibrillation was induced with a flicker frequency between 50 and $200 \mathrm{Hertz}(\mathrm{Hz})$. After ECG-confirmed ventricular fibrillation and 5 minutes of no-flow time, basic life support was started with mechanical chest compressions by the LUCAS 2-System (Stryker, Kalamazoo, $\mathrm{MI}$, USA) with a frequency of 100 compressions/min. Ventilation was performed according to the intervention group. Following the trial protocol, animals were randomized into 6 intervention groups $(n=7$ per group): IPPV PEEP 0 mbar (I0), IPPV PEEP 8 mbar (I8), IPPV PEEP 16 mbar (116), ULTVV PEEP 0 mbar (U0), ULTVV PEEP 8 mbar (U8), and ULTVV PEEP 16 mbar (U16) (Table 1).

After 30 minutes of basic life support (BLS), a rhythm analysis was performed, and guideline-based advanced life support (ALS) was applied if ventricular fibrillation was still detectable. At the CPR measured timepoints of $5 \mathrm{~min}, 15 \mathrm{~min}$ and $25 \mathrm{~min}$, samples for arterial and central venous blood gas analysis and MIGET measurements were taken. The extended hemodynamic measurements were recorded continuously by using the Datex Ohmeda S5 monitor (GE Healthcare, Munich, Germany). EIT loops were recorded continuously during CPR.

Postmortem lung tissue samples were collected from the cranial, caudal, ventral, and dorsal sections of the left and right lung lobes and fixed with formalin $4 \%$. These samples were paraffinized, cut into 2 micrometer thick slices and stained with hematoxylin eosin (HE) by the tissue bank of the University Medical Center Mainz.

\section{Scores and statistics}


The histopathologic lung samples were examined with an Olympus microscope (CX43RF, Olympus Cooperation, Tokyo Japan) via CellSens Software (CellSens Entry.Ink, creation date 03.12.2018) and scored with the previously established DAD-Score, which includes seven items: overdistension, epithelial destruction, inflammatory infiltration, alveolar and interstitial edema, hemorrhage and microatelectasis (13). Each item per sample was graded according to its severity from zero to five points in four nonoverlapping fields of view. In a global overview, the severity of each item for the entire region was also evaluated from zero to five points. The highest possible score was 175 points.

All statistical planning and interpretations were performed with the assistance of the Institute of Medical Biometrics and Epidemiology of the Johannes Gutenberg University Mainz. Statistical analyses were performed with SPSS (IBM SPSS Statistics, Version: 23 V 5 R, USA) by using repeated measurements of ANOVA (RMA) and post hoc analysis with Tukey's test. Statistics of the DAD score were evaluated using linear mixed-effect models. Data in text and graphs are presented as the mean and standard deviation (SD). $P$ values lower than 0.05 were considered statistically significant.

\section{Results}

42 experiments were performed in which no animal achieved a return of spontaneous circulation (ROSC). The driving pressure (Pdrive) showed a significant difference between the PEEP groups (RMA $p<0.001$ ), when adjusting for the ventilation mode, PEEP $16 \mathrm{mbar}$ had significantly lower values than PEEP $8 \mathrm{mbar}$ (Tukey $p=0.045$ ) and PEEP 0 mbar (Tukey $p<0.001$ ) during CPR. PEEP 8 mbar also showed lower values than PEEP 0 mbar (Tukey $p=0.014$ ). The comparison of the six intervention groups showed analogous findings (Tukey 10 vs. 116, $p=0.010$; Tukey U0 vs. U16, $p=0.003$ ). PEEP 16 displayed a marginal mean Pdrive of $12,23 \mathrm{mbar}( \pm 5,04 \mathrm{mbar}$ ), while PEEP 0 mbar showed a marginal mean Pdrive of $21.06 \mathrm{mbar}$ ( \pm 5,91 mbar). The PEEP groups also showed significant differences when observing the mean

(Pmean) (RMA p < 0.001) and peak pressure (Ppeak) (RMA p<0.001) during CPR. PEEP 0 and 8 mbar had significantly lower values than PEEP 16 mbar in Pmean (Tukey PEEP 0 mbar vs. PEEP 16 mbar, $p<0.001$; Tukey PEEP 8 mbar vs. PEEP 16 mbar, $p<0.001$; Tukey PEEP 0 mbar vs. PEEP 8 mbar, $p<0.001$ ) and Ppeak (Tukey PEEP 0 mbar vs. PEEP 16 mbar, $p<0.001$; Tukey PEEP 8 mbar vs. PEEP 16 mbar, $p=0.031$ ). Similar findings may be observed when comparing the Pmean of the intervention groups. In Ppeak the intervention groups showed a significant difference when comparing 10 vs. 116 (Tukey, $p=0.011$ ). PEEP 16 mbar displayed a marginal mean Ppeak of 28,49 mbar ( \pm 5,28 mbar), while PEEP 0 mbar showed a marginal mean Ppeak of 21.23 mbar ( $\pm 5,89$ mbar). Additionally, significant differences were observed when comparing the ventilation modes, adjusted for the PEEP groups, in the Pdrive (RMA $p=0.002$ ), Pmean (RMA $p<0.001)$ and Ppeak (RMA $p=0.001)$ parameters during $C P R$, with IPPV leading to significantly higher values than ULTVV (Figure 1).

The V/Q were measured via MIGET. The UO group showed nonsignificant higher percentages of shunt and significantly higher percentages of low $\mathrm{V} / \mathrm{Q}$ (Tukey $10 \mathrm{vs}$. $\mathrm{U} 0, \mathrm{p}=0.038$ ) as well as a nonsignificant lower fraction of normal and high V/Q during CPR. The 10 group showed nonsignificant increasing normal V/Q as well as high V/Q during the intervention and decreased shunt percentage. Both PEEP 16 
mbar groups showed decreasing normal V/Q percentages during the intervention and increasing high and low V/Q as well as shunt percentages. The ULTVV groups showed significantly fewer high V/Q (RMA $p=0.006)$ while having significantly more results of low $V / Q(R M A p=0.041)$, adjusted for the PEEP groups.

The arterial partial pressure of carbon dioxide (paCO2) was significantly higher in the ULTVV mode during the entire intervention (RMA $\mathrm{p}=0.001$ ), when adjusted for PEEP. At the start of the intervention, the PEEP 0 mbar groups and the U8 group showed high values of paCO2 while at the end, the groups with PEEP 16 mbar displayed the highest paCO2 values. The significantly lowest arterial partial pressure of oxygen (paO2) was detected in the PEEP 0 mbar group, adjusted for the ventilation mode (Tukey PEEP 0 mbar vs. PEEP 8 mbar, $p=0.025)$. In all intervention groups, the paO2 decreased over time (Figure 2).

There were no significant differences in hemodynamics between the PEEP groups or tidal volume groups. However, UO mbar showed a nonsignificant lower mean arterial pressure (MAP) during CPR than the groups with PEEP (Figure 3). A detailed summary of cardiopulmonary parameters is shown in table 2. The lung physiology was monitored via EIT. In the resulting transverse sectional view, the regions of interests (ROIs) are numbered 1 to 4 from the ventral thoracic areas (1) to the dorsal areas (4). During CPR, the highest impedances were observed in ROI 2.

In all ROIs, no significant differences were found between the groups during the intervention. In ROI 1, U0 had nonsignificant increased impedances compared with the two ULTTV groups with PEEP. In the dorsal thoracic part, the ULTVV mode displayed nonsignificantly higher impedances than the IPPV mode when adjusted for the PEEP groups. Here, UO showed high impedances at the beginning of CPR, which then constantly decreased over time. The highest values in the dorsal thoracic part were observed in the PEEP 16 mbar groups (Figure 4).

Lung histology was evaluated with the DAD-score. There was significantly higher lung damage in the sum total category in the PEEP 0 mbar group (all DAD score-associated significances were evaluated by linear mixed-effect models) (vs. PEEP 8 mbar, $p=0.038$; vs. PEEP $16 \mathrm{mbar}, \mathrm{p}=0.009$ ), which could also be observed when comparing the IPPV intervention groups (I0 vs. I8, $p=0.012 ; 10$ vs. I16, $p=0.040$ ). Nonetheless, the IPPV group showed lower values than the ULTVV group (IPPV vs. ULTVV, $p=0.012$ ), which was also observed in an intervention group comparison (I8 vs. U8, $p=0.003$ ). Regarding the individual items of the DAD score, the ULTVV mode, adjusted for the PEEP groups, showed greater microatelectrauma (vs. IPPV, $p<0.001$ ), while the IPPV group showed more overdistension (vs. ULTVV, $p=$ 0.001). In examining the PEEP groups, both PEEP 16 mbar groups showed the greatest overdistension within their ventilation mode (U16 vs. U0, $\mathrm{p}<0.001 ; \mathrm{U} 16$ vs. U8, $\mathrm{p}=0.001$ ). The PEEP 0 mbar groups showed the most microatelectatic tissue in their ventilation mode (I0 vs. I8, $p=0.016$; U0 vs. U16, $p=0.013)$ as well as hemorrhage (I0 vs. I8, $p=0.049$; U0 vs. U8, $p=0.013$, U0 vs. U16, $p<0.001$ ) (Figure 5). Microphotographs are shown in Figure 6.

\section{Discussion}


In this prospective randomized controlled animal trial, we examined three different PEEP-levels in a standard and low tidal volume ventilation mode during CPR. We discovered that the application of higher PEEP values significantly decrease driving pressures, when adjusted for the ventilation mode. Additionally, we showed that increased PEEP levels did not substantially impair mean arterial blood pressure levels during CPR, suggesting a general feasibility while resuscitation efforts via chestcompressions are ongoing. EIT and MIGET measurements supported the hypothesis, that higher PEEP provides improved recruitment of dependent lung areas, whereas 0 mbar PEEP showed higher histologic damage values with increased atelectrauma.

Ventilation during resuscitation is challenging because of extreme thoracic pressure variances due to chest compressions. The concept of lung protective ventilation recommends using PEEP greater than 5 $\mathrm{cm} \mathrm{H} 2 \mathrm{O}$, maintaining Ppeak below $30 \mathrm{~cm} \mathrm{H} 2 \mathrm{O}$ and aiming for a low Pdrive, limiting it to $15 \mathrm{~cm} \mathrm{H} 2 \mathrm{O}$ or less $(12,14)$. In this trial, the intervention groups with PEEP showed significantly lower Pdrive values during CPR compared to PEEP 0 mbar. When comparing IPPV versus ULTVV, the latter showed significantly lower peak and driving pressures in the respective PEEP groups. This is expected considering that the volume-controlled ventilation mode is set to substantially lower volumes initially. Lower tidal volume strategies lead to less alveolar pressure and thus can avoid overdistention (15). The reduction of high ventilation pressures can lower the risk of overdistension, which can cause harmful lung damage and may lead to barotrauma $(11,12,15)$.

The histologic examination of both the PEEP 16 mbar groups and all three IPPV groups revealed more overdistended lung tissue, suggesting overinflation and increased stress caused by the increased ventilation pressures necessary to achieve the set tidal volumes. However, the overall lung damage was substantial in the ULTVV mode and the PEEP 0 mbar group, which was mainly driven by significantly higher ratios of bleeding and microatelectasis. This leads to the question of which type of tissue damage - if any - is more crucial for post-ROSC oxygenation and overall outcome. To the best of our knowledge, no concise data are available on this correlation. Using PEEP in general can be beneficial because it leads to better oxygenation and lung recruitment as well as less atelectatic tissue (16). Atelectasis can lead to impaired systemic oxygenation $(4,6)$. The paO2 values decreased in all six intervention groups during the intervention. However, when using PEEP, increased oxygenation could be observed in this trial. A study that investigated whether different PEEP levels could optimize carbon dioxide clearance during CPR showed that higher PEEP levels lead to significantly decreased paCO2 levels and increased minute volume because of a higher fraction of gas oscillations generated by chest compressions (17). However, this study intentionally did not adhere to resuscitation guidelines regarding respiratory rates, partially explaining those results and potentially reducing their clinical relevance. In our trial, at early resuscitation timepoints, the paCO2 was high in the groups without PEEP and the U8 group. The results of the MIGET measurements support the observation of impaired gas exchange when not using PEEP. Especially in the UO group, overall global hypoventilation with higher shunt perfusion was found. Determinations of high, normal, and low V/Q ratios and shunt volumes were achieved by analyzing gas elimination during lung perfusion using a mass spectrometer. The use of MIGET technology during CPR was validated in previous trials $(7,18)$ and can provide additional information about (impaired) circulation and ventilation 
during CPR. In the IPPV group, a distinct increase in high V/Q volume was observed compared to ULTVV when adjusted for PEEP, which supports an increase in hyperinflated lung areas and is in accordance with previous studies (7). When using PEEP, lower shunt fractions could be detected, particularly in early resuscitation, suggesting improved recruitment and optimized ventilation. This aligns with studies that showed that using PEEP during resuscitation can prevent airway closure and ensure alveolar ventilation $(19,20)$ and has positive effects on systemic oxygenation $(4,5,21)$. In our trial, oxygenation was improved when using PEEP, especially in early resuscitation. The ameliorated oxygenation could result from the prevention of atelectasis $(4,6,7)$, thus decreasing shunt perfusion, improving lung recruitment and preventing airway closure $(19,20)$. The EIT recordings showed normo- to hyperventilated lung areas when using PEEP, especially when ventilating with IPPV. EIT measurements during CPR have not yet been systematically performed and can only yield supportive data, even though general feasibility has been shown previously (22). Generally, EIT can support the titration of PEEP to avoid atelectasis and prevent regional hyperinflation (23-26). In a direct comparison of not using PEEP versus using PEEP, the use of PEEP leads to the redistribution of ventilation from the ventral to the dorsal lung regions (8), which could also be suspected in our recordings during CPR, especially in the PEEP 16 groups. Further experimental assessment is needed to validate the method during CPR.

In terms of prolonged CPR, all PEEP groups showed decreasing paO2 with an increase in shunt perfusion and lower normal V/Q values. These effects were less pronounced in the PEEP 8 groups, which could emphasize the beneficial effects of moderate PEEP for longer use of CPR.

Interestingly, even when using high PEEP levels, we found no differences in the hemodynamic values although theoretically, a continuous application of PEEP and a consecutive high Ppeak and Pmean could lead to increased intrathoracic pressure which could cause impaired venous return and, consequently, cardiac output (CO) $(10,11,21)$. A study evaluating the effects of PEEP on CO during CPR suggested a PEEP of 5 mbar as the optimal level in their model (21). However, the detected decreases - albeit statistically significant - were marginal in absolute values, showing a CO decrease of $0.3 \mathrm{~L} / \mathrm{min}$ when comparing a PEEP of 0 mbar with 20 mbar, thus determining that the clinical relevance in resuscitation is uncertain. This also aligns with the findings of the present study concerning the MAP, where no significant differences could be detected, which is in line with previous studies focusing on the ULTVV and IPPV modes $(7,27)$, even if only a PEEP of 5 mbar was used in these studies.

The use of adrenaline has been shown to increase the rates of survival to hospital admission and longterm survival, although there was no significant differences in cases that a favorable neurologic outcome was observed $(2,28,29)$. However, because our trial did not have the specific goal of ROSC, no vasopressors were used during CPR as recommended by the guidelines $(2,3)$. While there were no differences in MAP when using PEEP, potential effects on survival, neurological outcome and pulmonary function should be examined in further studies with a focus on advanced life support algorithms and post-CPR monitoring.

\section{Conclusion}


This prospective randomized controlled animal trial showed that the use of PEEP during CPR ventilation seems beneficial. It leads to a ventilation pattern with lower driving pressures, optimized ventilationperfusion ratios, lower shunt perfusion as well as less atelectatic lung tissue and less overall lung damage. Additionally, no detrimental hemodynamic effects were observed even with high PEEP levels, emphasizing the potential benefits of oxygenation in a ROSC setting without compromising organ perfusion. Further studies are needed to confirm these results and to examine potential long-term effects in resuscitated animals.

\section{Abbreviations}

AHA American Heart Association

ALS advanced life support

BLH baseline healthy

BLS basic life support

CO cardiac output

CPR cardiopulmonary resuscitation

DAD Score diffuse alveolar damage score

EIT electric impedance tomography

etCO2 end-expiratory $\mathrm{CO} 2$

ERC European Resuscitation Council

HE hematoxylin eosin

IPPV intermittent positive pressure ventilation

10

IPPV PEEP 0 mbar

18

IPPV PEEP 8 mbar

116

IPPV PEEP 16 mbar

MAP mean arterial pressure

MMIMS-MIGET micropore membrane inlet mass spectrometry facilitated multipleinert gas elimination technique 


$\begin{array}{lc}\text { paCO2 } & \text { arterial partial pressure of carbon dioxide } \\ \text { paO2 } & \text { arterial partial pressure of oxygen } \\ \text { Pdrive } & \text { driving pressure } \\ \text { Peep } & \text { positive end-expiratory pressure } \\ \text { Pmean } & \text { mean pressure } \\ \text { Ppeak } & \text { peak pressure } \\ \text { SD } & \text { standard deviation } \\ \text { RMA } & \text { repeated measurements of ANOVA } \\ \text { ROI } & \text { region of interest } \\ \text { ROSC } & \text { return of spontaneous circulation } \\ \text { ULTVV } & \text { ultralow tidal volume ventilation } \\ \text { U0 } & \text { ULTVV PEEP } 0 \text { mbar } \\ \text { U8 } & \text { ULTVV PEEP 8 mbar } \\ \text { U16 } & \text { ULTVV PEEP 16 mbar } \\ \text { Vt } & \text { tidal volume } \\ \text { V/Q } & \text { Ventilation/perfusion }\end{array}$

\section{Declarations}

Consent for publication

Not applicable

\section{Availability of data and materials}

All data analyzed for this study are provided in the manuscript.

\section{Competing interests}

The authors declare that they have no competing interests.

Ethics approval and consent to participate 
This animal trial was approved by the State and Institutional Animal Care Committee Rhineland Palatine (approval no. G20-1-065) and all experiments were performed according to the German Animal Protection Law following the ARRIVE guidelines.

\section{Funding}

This study was partially funded by a personal grant of the German Research Foundation to Robert Ruemmler (DFG grant no.: RU 2371/1-1). No additional external funding was used. The LUCAS-2 device was provided by the manufacturer unconditionally.

\section{Author contributions}

EKH, MR and RRu designed and planned the study, MR, LM, JR, KM and Rri performed the experiments, MR, AZ and Rru analyzed the data, LM performed histologic analyses, MR, Rru and EKH drafted the manuscript.

\section{Acknowledgments}

This trial will be part of the doctoral thesis to the Johannes Gutenberg-University Mainz written by Leah Müllejans. We would like to thank D. Dirvonskis for her committed and active support during the entire trial. The LUCAS 2-system and the EIT device were provided unconditionally by the manufacturers for animal research purposes only. Tissue samples were provided by the tissue bank of the University Medical Center Mainz in accordance with the regulations of the tissue biobank and the approval of the ethics committee of the University Medical Center Mainz.

\section{References}

1. Newell C, Grier S, Soar J: Airway and ventilation management during cardiopulmonary resuscitation and after successful resuscitation. Crit Care 2018, 22(1):190.

2. Soar J, Bottiger BW, Carli P, Couper K, Deakin CD, Djarv T, Lott C, Olasveengen T, Paal P, Pellis T et al: European resuscitation council guidelines 2021: adult advanced life support. Resuscitation 2021, 161:115-151.

3. Panchal AR, Bartos JA, Cabanas JG, Donnino MW, Drennan IR, Hirsch KG, Kudenchuk PJ, Kurz MC, Lavonas EJ, Morley PT et al: Part 3 adult basic and advanced life support: 2020 American Heart Association guidelines for cardiopulmonary resuscitation and emergency cardiovascular care. Circulation 2020, 142(16_suppl_2):S366-S468.

4. McCaul C, Kornecki A, Engelberts D, McNamara P, Kavanagh BP: Positive end-expiratory pressure improves survival in a rodent model of cardiopulmonary resuscitation using high-dose epinephrine. Anesth Analg 2009, 109(4):1202-1208.

5. Hodgkin BC, Lambrew CT, Lawrence FH, 3rd, Angelakos ET: Effects of PEEP and of increased frequency of ventilation during CPR. Crit Care Med 1980, 8(3):123-126. 
6. Kill C, Hahn O, Dietz F, Neuhaus C, Schwarz S, Mahling R, Wallot P, Jerrentrup A, Steinfeldt T, Wulf H et al: Mechanical ventilation during cardiopulmonary resuscitation with intermittent positive-pressure ventilation, bilevel ventilation, or chest compression synchronized ventilation in a pig model. Crit Care Med 2014, 42(2):e89-e95.

7. Ruemmler R, Ziebart A, Moellmann C, Garcia-Bardon A, Kamuf J, Kuropka F, Duenges B, Hartmann EK: Ultra-low tidal volume ventilation-a novel and effective ventilation strategy during experimental cardiopulmonary resuscitation. Resuscitation 2018, 132:56-62.

8. Frerichs I, Schmitz G, Pulletz S, Schadler D, Zick G, Scholz J, Weiler N: Reproducibility of regional lung ventilation distribution determined by electrical impedance tomography during mechanical ventilation. Physiol Meas 2007, 28(7):S261-S267.

9. Shono A, Katayama N, Fujihara T, Bohm SH, Waldmann AD, Ugata K, Nikai T, Saito Y: Positive endexpiratory pressure and distribution of ventilation in pneumoperitoneum combined with steep trendelenburg position. Anesthesiology 2020, 132(3):476-490.

10. Richard JC, Lefebvre JC, Tassaux D, Brochard L: Update in mechanical ventilation 2010. Am J Respir Crit Care Med 2011, 184(1):32-36.

11. Pinsky MR, Summer WR, Wise RA, Permutt S, Bromberger-Barnea B: Augmentation of cardiac function by elevation of intrathoracic pressure. J Appl Physiol Respir Environ Exerc Physiol 1983, 54(4):950-955.

12. Fichtner F, Moerer O, Weber-Carstens S, Nothacker M, Kaisers U, Laudi S, Guideline g: Clinical guideline for treating acute respiratory insufficiency with invasive ventilation and extracorporeal membrane oxygenation: evidence-based recommendations for choosing modes and setting parameters of mechanical ventilation. Respiration 2019, 98(4):357-372.

13. Ziebart A, Hartmann EK, Thomas R, Liu T, Duenges B, Schad A, Bodenstein M, Thal SC, David M: Low tidal volume pressure support versus controlled ventilation in early experimental sepsis in pigs. Respir Res 2014, 15:101.

14. Amato MB, Meade MO, Slutsky AS, Brochard L, Costa EL, Schoenfeld DA, Stewart TE, Briel M, Talmor $\mathrm{D}$, Mercat A et al: Driving pressure and survival in the acute respiratory distress syndrome. $N$ Engl $J$ Med 2015, 372(8):747-755.

15. Cheifetz IM, Craig DM, Quick G, McGovern JJ, Cannon ML, Ungerleider RM, Smith PK, Meliones JN: Increasing tidal volumes and pulmonary overdistention adversely affect pulmonary vascular mechanics and cardiac output in a pediatric swine model. Crit Care Med 1998, 26(4):710-716.

16. Acosta P, Santisbon E, Varon J: "The use of positive end-expiratory pressure in mechanical ventilation". Crit Care Clin 2007, 23(2):251-261, x.

17. Levenbrown Y, Hossain MJ, Keith JP, Burr K, Hesek A, Shaffer TH: Effect of positive end-expiratory pressure on additional passive ventilation generated by CPR compressions in a porcine model. Intensive Care Med Exp 2021, 9(1):37.

18. Hartmann EK, Duenges B, Boehme S, Szczyrba M, Liu T, Klein KU, Baumgardner JE, Markstaller K, David M: Ventilation/perfusion ratios measured by multiple inert gas elimination during experimental 
cardiopulmonary resuscitation. Acta Anaesthesiol Scand 2014, 58(8):1032-1039.

19. Charbonney E, Grieco DL, Cordioli RL, Badat B, Savary D, Richard JM, Group C: Ventilation during cardiopulmonary resuscitation: what have we learned from models? Respir Care 2019, 64(9):11321138.

20. Cordioli RL, Grieco DL, Charbonney E, Richard JC, Savary D: New physiological insights in ventilation during cardiopulmonary resuscitation. Curr Opin Crit Care 2019, 25(1):37-44.

21. Levenbrown Y, Hossain MJ, Keith JP, Burr K, Hesek A, Shaffer T: The effect of positive end-expiratory pressure on cardiac output and oxygen delivery during cardiopulmonary resuscitation. Intensive Care Med Exp 2020, 8(1):36.

22. Kamuf J, Garcia-Bardon A, Duenges B, Liu T, Jahn-Eimermacher A, Heid F, David M, Hartmann EK: Endexpiratory lung volume measurement correlates with the ventilation/perfusion mismatch in lung injured pigs. Respir Res 2017, 18(1):101.

23. Bodenstein M, David M, Markstaller K: Principles of electrical impedance tomography and its clinical application. Crit Care Med 2009, 37(2):713-724.

24. Muders $\mathrm{T}$, Luepschen $\mathrm{H}$, Putensen $\mathrm{C}$ : Impedance tomography as a new monitoring technique. Curr Opin Crit Care 2010, 16(3):269-275.

25. Luepschen $\mathrm{H}$, Meier T, Grossherr M, Leibecke T, Karsten J, Leonhardt S: Protective ventilation using electrical impedance tomography. Physiol Meas 2007, 28(7):S247-S260.

26. Walsh BK, Smallwood CD: Electrical impedance tomography during mechanical ventilation. Respir Care 2016, 61(10):1417-1424.

27. Ruemmler R, Ziebart A, Kuropka F, Duenges B, Kamuf J, Garcia-Bardon A, Hartmann EK: Bi-Level ventilation decreases pulmonary shunt and modulates neuroinflammation in a cardiopulmonary resuscitation model. PeerJ 2020, 8:e9072.

28. Perkins GD, Ji C, Deakin CD, Quinn T, Nolan JP, Scomparin C, Regan S, Long J, Slowther A, Pocock H et al: A randomized trial of epinephrine in out-of-hospital cardiac arrest. N Engl J Med 2018, 379(8):711-721.

29. Panchal AR, Berg KM, Hirsch KG, Kudenchuk PJ, Del Rios M, Cabanas JG, Link MS, Kurz MC, Chan PS, Morley PT et al: 2019 American Heart Association focused update on advanced cardiovascular life support: use of advanced airways, vasopressors, and extracorporeal cardiopulmonary resuscitation during cardiac arrest: an update to the American Heart Association guidelines for cardiopulmonary resuscitation and emergency cardiovascular care. Circulation 2019, 140(24):e881e894.

\section{Tables}

Table 1. Group design and intervention parameters during resuscitation. Overview of relevant ventilation parameters, invasively measured hemodynamic parameters, blood gases, MIGET measurement, DADScore. Standard deviation (SD), driving pressure $\left(P_{\text {drive }}\right)$, peak pressure $\left(P_{\text {peak }}\right)$, partial arterial pressure of 
carbon dioxide $\left(\mathrm{paCO}_{2}\right)$, partial arterial pressure of oxygen $\left(\mathrm{paO}_{2}\right)$, mean arterial pressure (MAP), diffuse alveolar damage (DAD)-score, overdistension (overdis.), intermittent positive pressure ventilation (IPPV), ultralow tidal volume ventilation (ULTVV). Statistical analyses were performed using SPSS and p-values $<0.05$ were considered significant.

\begin{tabular}{|lll|}
\hline Group parameters & Group 1-3 & Group 4-6 \\
\hline Ventilation mode & IPPV & ULTVV \\
\hline PEEP Level & $0,8,16 \mathrm{mbar}$ & $0,8,16 \mathrm{mbar}$ \\
\hline Tidal volume $(\mathrm{Vt})$ & $9-10 \mathrm{ml} / \mathrm{kgBW}$ & $2-3 \mathrm{ml} / \mathrm{kgBW}$ \\
\hline Respiratory rate $(\mathrm{RR})$ & $10 \mathrm{breaths} / \mathrm{min}$ & $50 \mathrm{breaths} / \mathrm{min}$ \\
\hline $\mathrm{FiO}_{2}$ & 1.0 & 1.0 \\
\hline
\end{tabular}

Table 2 is only available as a download in the Supplemental Files section.

\section{Figures}
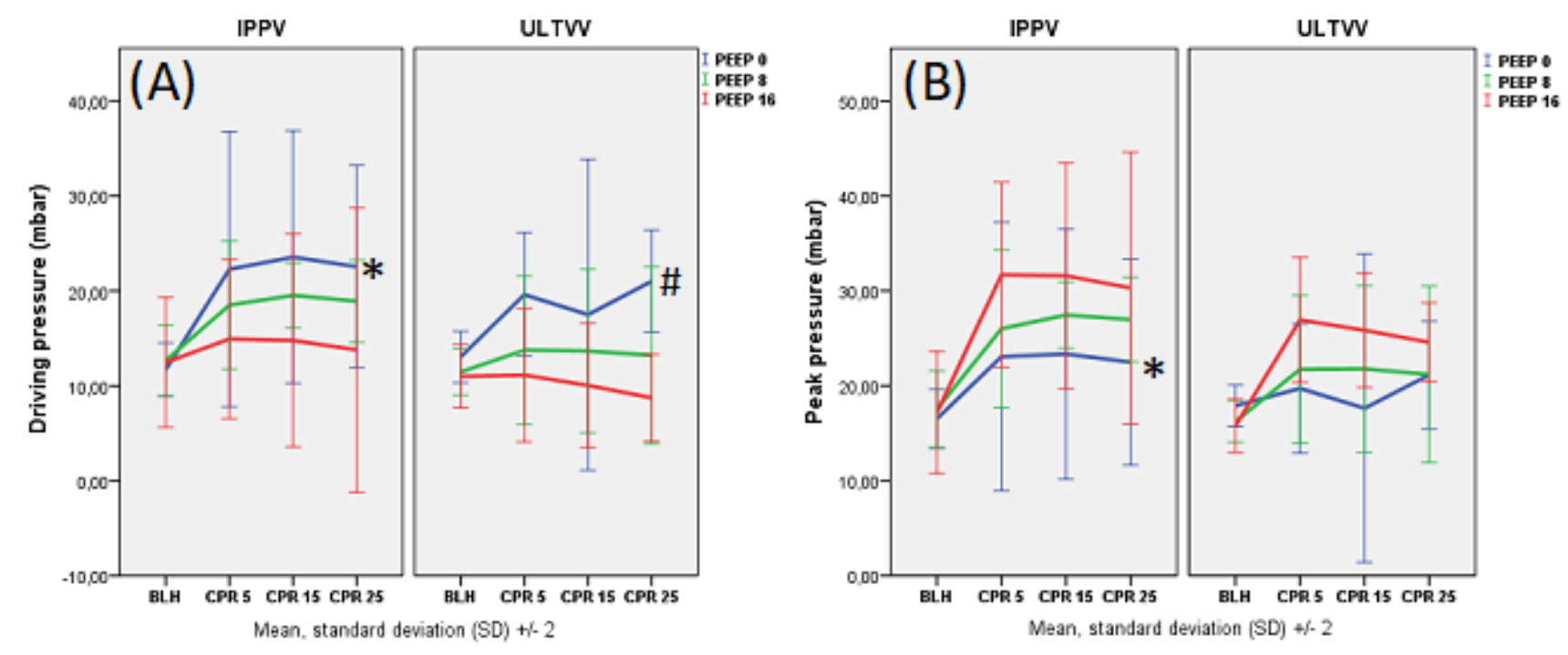

\section{Figure 1}

Ventilation: driving pressure (Pdrive, A), peak pressure (Ppeak, B). Airway pressures were measured in mbar. Data are shown as mean values and standard deviation (SD). Significant differences in Pdrive (A): * vs. 116, $p=0.010$; \# vs. U16, $p=0.003$ (Tukey). Significant differences in Ppeak (B) * vs. 116, $p=0.011$ (Tukey). 

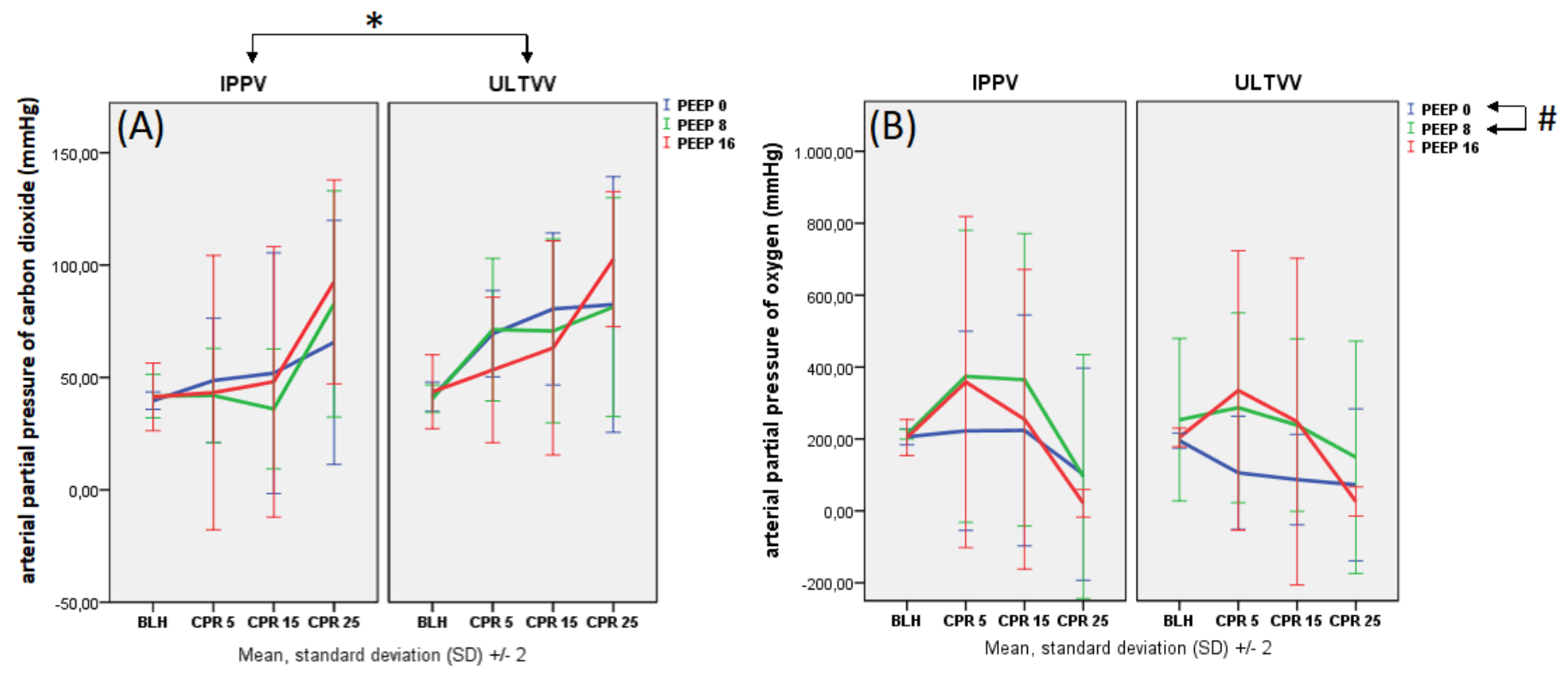

Figure 2

Blood gases: arterial partial pressure of $\mathrm{CO} 2(\mathrm{PaCO} 2, \mathrm{~A})$, arterial partial pressure of $\mathrm{O} 2(\mathrm{PaO} 2, \mathrm{~B})$. The unit of $\mathrm{PaCO} 2$ and $\mathrm{PaO} 2$ is $\mathrm{mmHg}$. Data are shown as mean values and standard deviation (SD). Significant differences in paCO2 (A): * ULTVV vs. IPPV, when adjusted for PEEP, $p=0.001$ (RMA). Significant differences in paO2 (B): \# PEEP 0 mbar vs. PEEP 8 mbar, when adjusted for ventilation mode, $p=0.025$ (Tukey). 


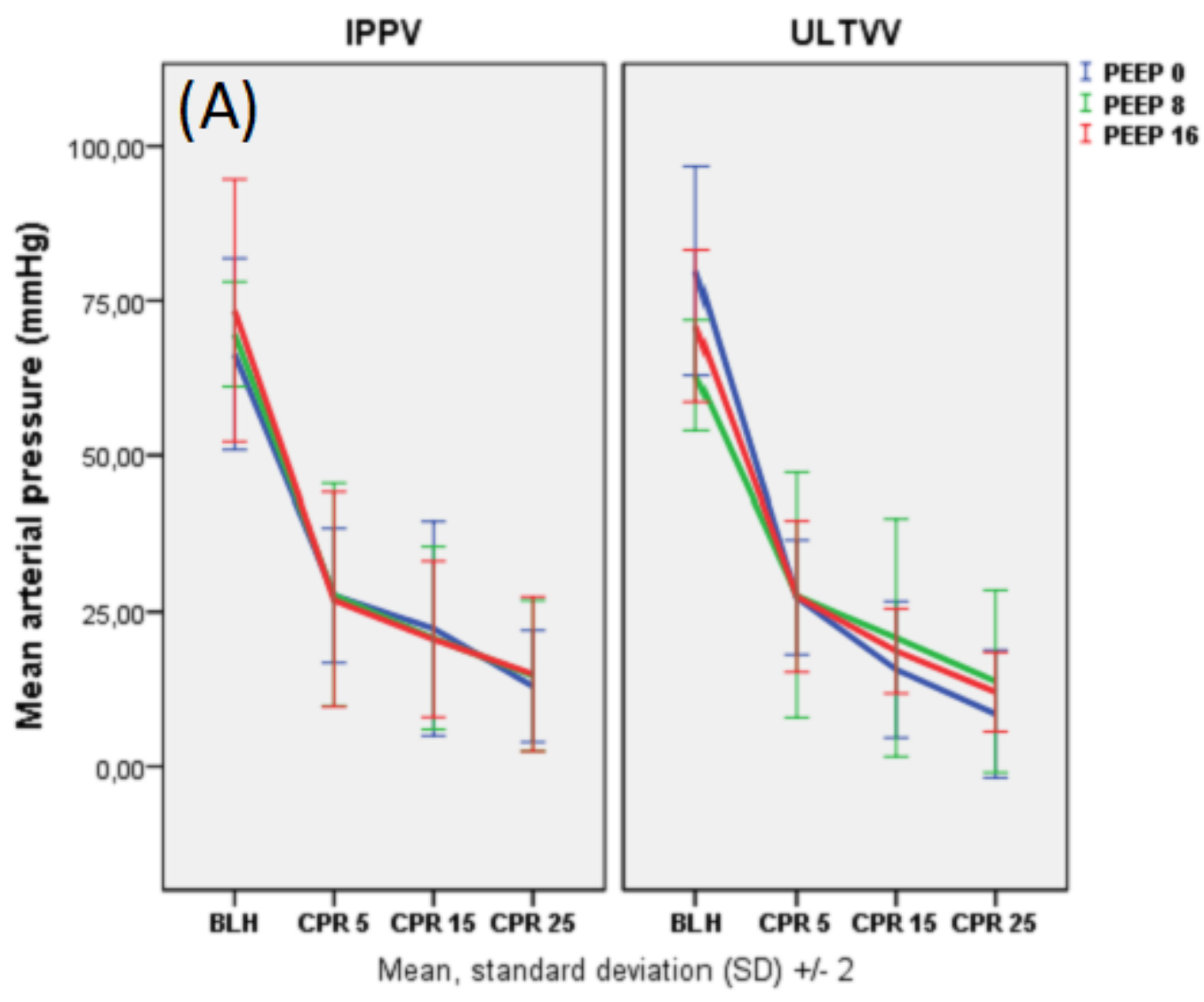

Figure 3

Hemodynamics: mean arterial pressure (MAP, A). The unit of MAP is measured in $\mathrm{mmHg}$. Data are shown as mean values and standard deviation (SD). No significant differences were observed. 
(A)

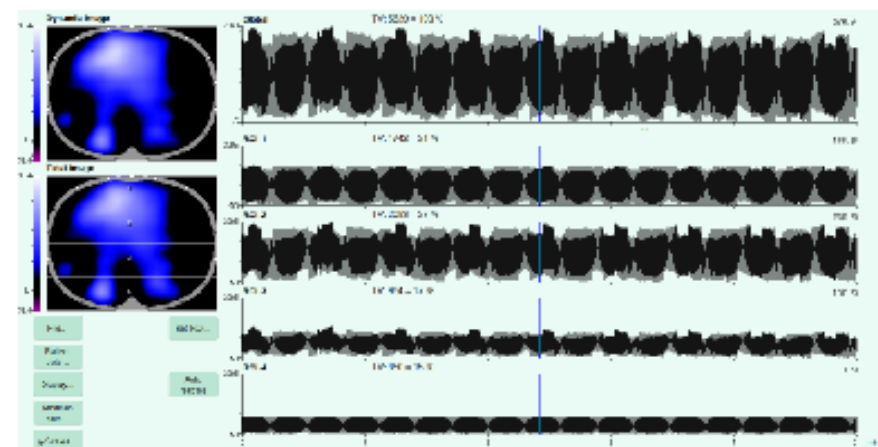

(C)

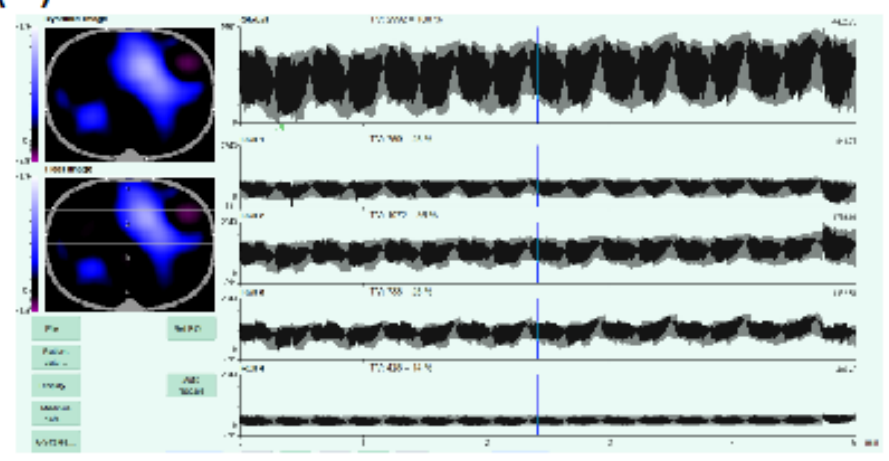

(B)

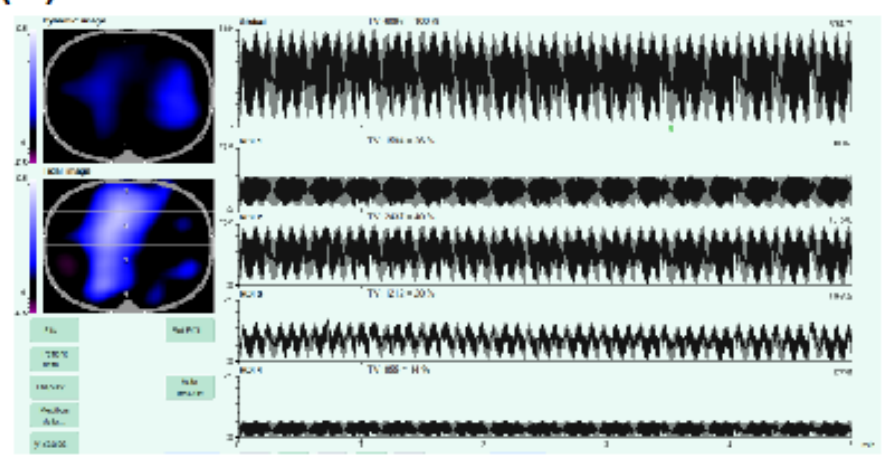

(D)

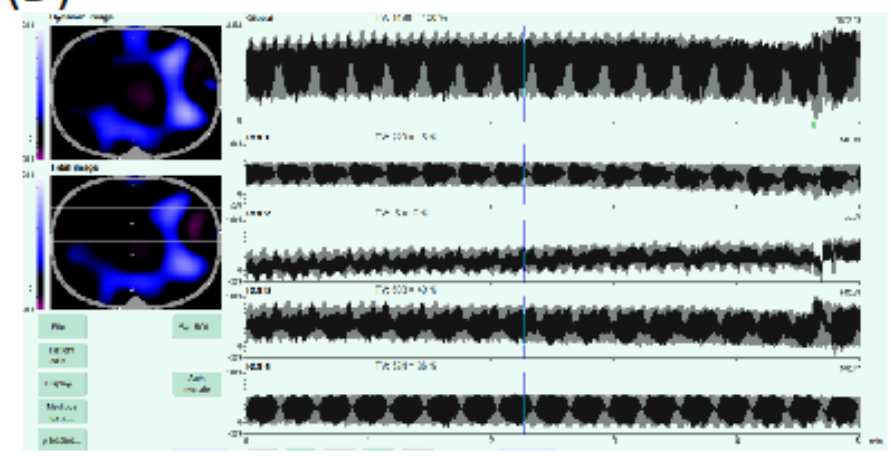

Figure 4

Lung physiology via Electrical impedance tomography (EIT): the picture shows EIT loops during CPR at the intervention timepoint CPR 5 min. $A=$ ULTVV PEEP 0 mbar, $B=I P P V$ PEEP 0 mbar, $C=$ ULTVV PEEP $16 \mathrm{mbar}, \mathrm{D}=$ IPPV PEEP 16 mbar. Global EIT recordings and recordings of ROI (region of interest) 1 to ROI 4 are pictured. There were no significant differences detected. 

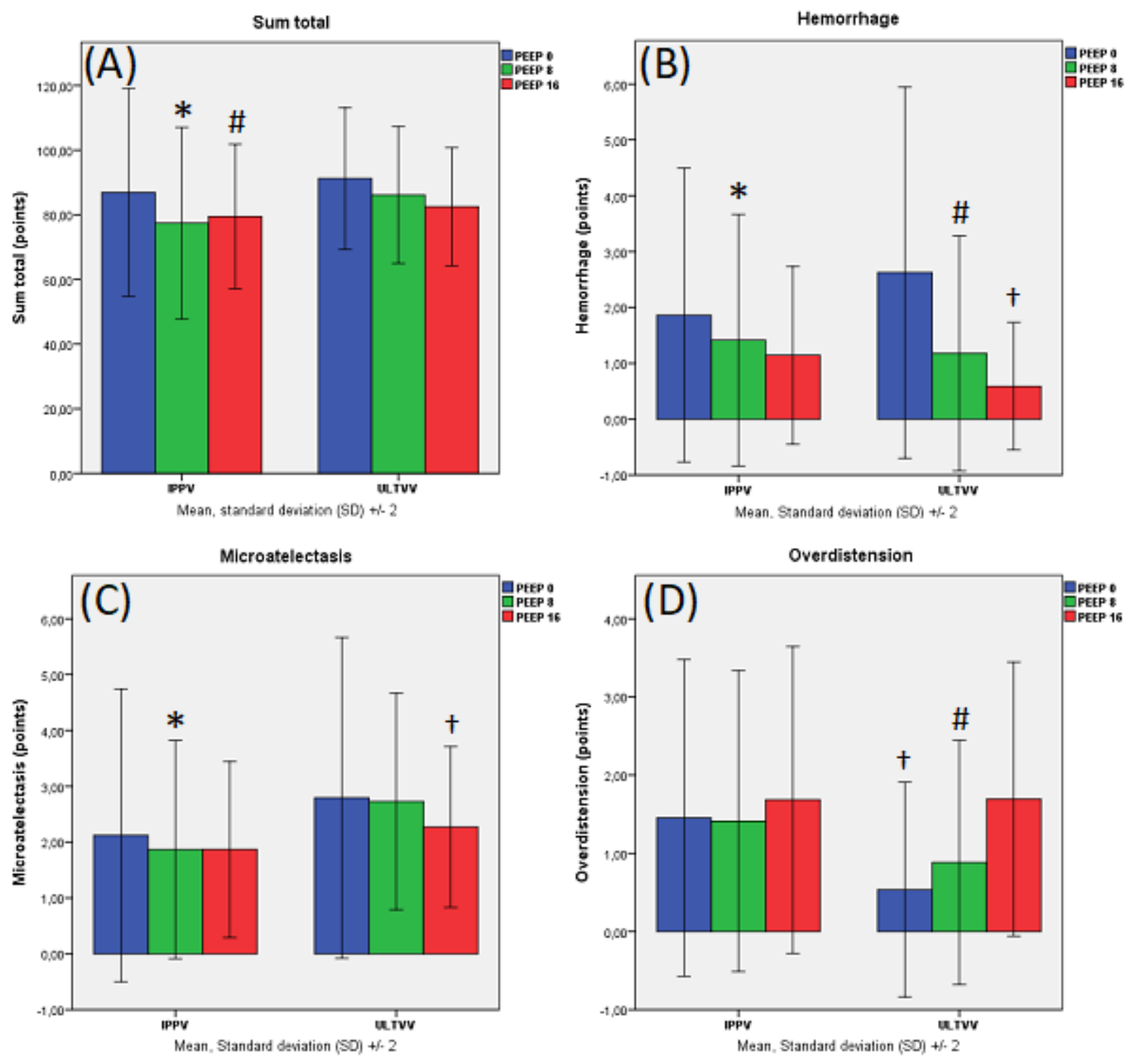

\section{Figure 5}

Lung histology evaluated with the DAD-score. Categories were scored in points. Data are shown as mean values and standard deviation (SD). Significant differences in sum total (A): * vs. I0, $p=0.012$; \# vs. I0, $p=0.040$ (linear mixed-effect models). Significant differences in hemorrhage (B): * vs. I0, $p=0.049$; \# vs. U0, $p=0.013 ; \dagger$ vs. U0, $p<0.001$ (linear mixed-effect models). Significant differences in microatelectasis (C): * vs. I0, $p=0.016$; † vs. U0, $p=0.013$ (linear mixed-effect models). Significant differences in overdistension (D): † vs. U16, $p<0.001$; \# vs. U16, p=0.001 (linear mixed-effect models). 
(A)

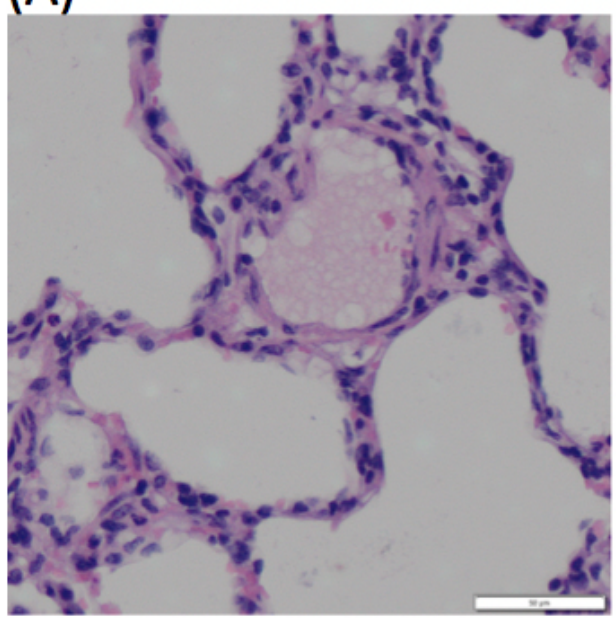

(D)

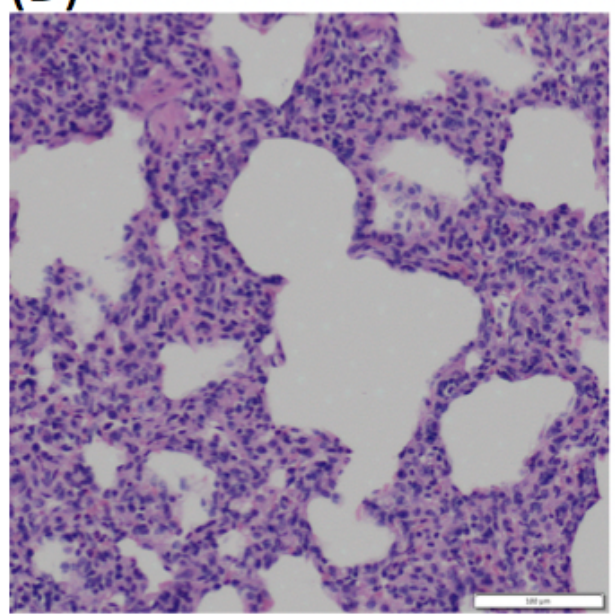

(G)

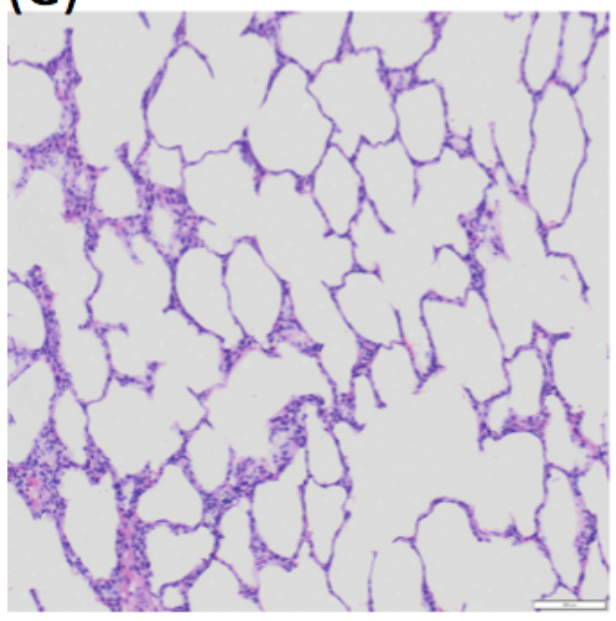

(B)

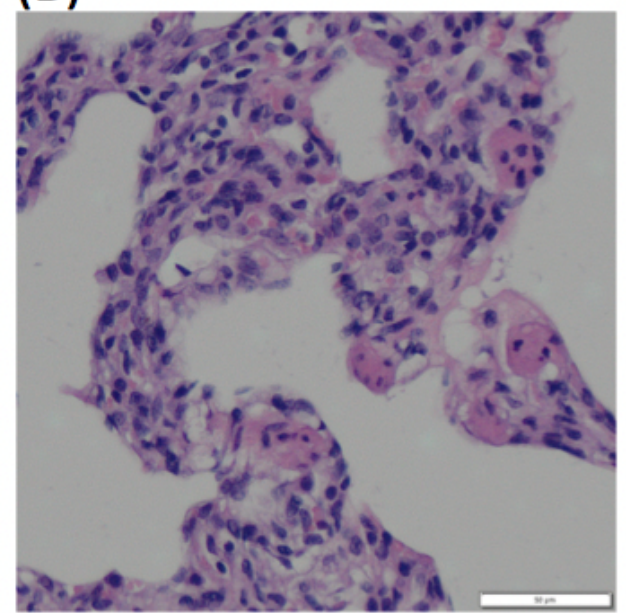

(E)

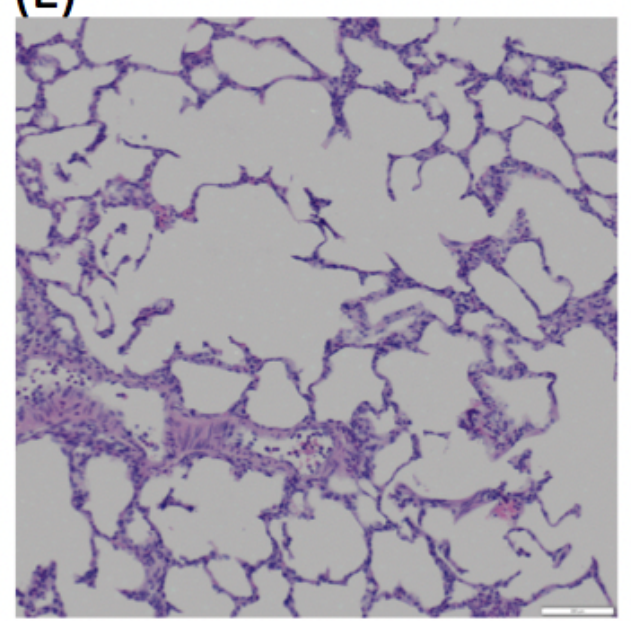

(C)

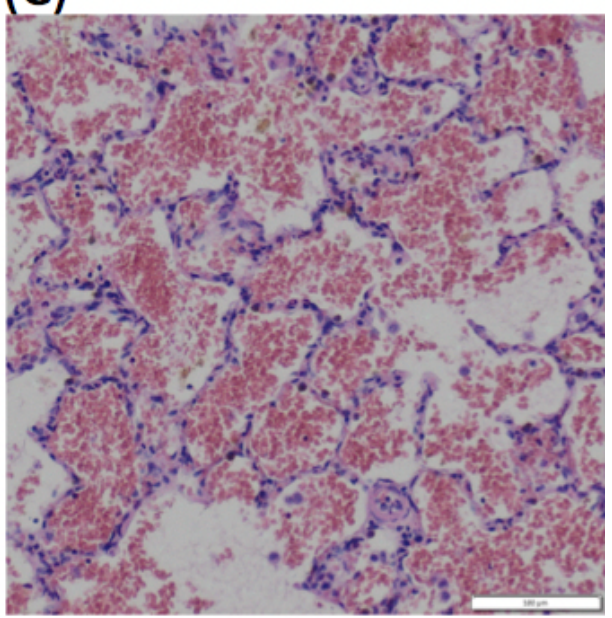

(F)

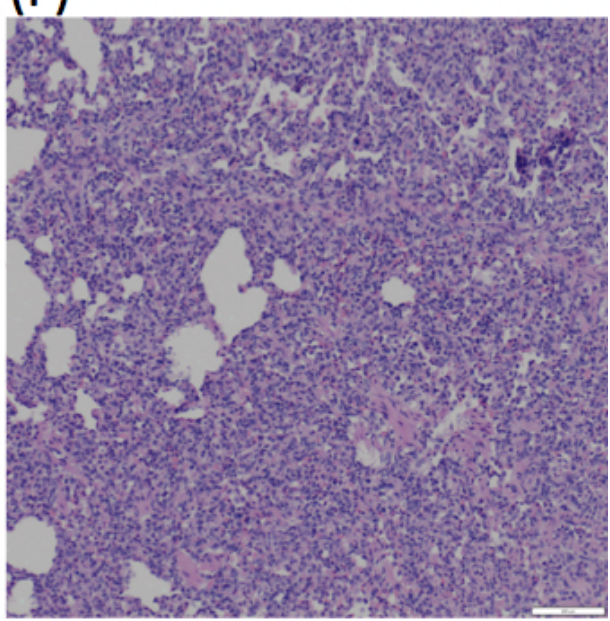

A: alveolar edema

B: interstitiell edema

A-B scale bars: scale bar $50 \mu \mathrm{m}$

C: hemmorrhage

D: inflammatory infiltration

E: epithelial destruction

$\mathrm{F}$ : microatelectasis

G: overdistension

C-G scale bars: $100 \mu \mathrm{m}$

\section{Figure 6}

Microphotographs of histologic lung samples with the 7 aspects of the DAD- Score used.

\section{Supplementary Files}

This is a list of supplementary files associated with this preprint. Click to download. 
- Table2.docx

Page 20/20 